\title{
High-resolution imaging in Espirito Santo basin through broadband processing, Full Waveform Inversion and Least Squares Migration
}

Guilherme Jangelme, Alejandro Alcudia-Leon; PGS

Copyright 2021, SBGf - Sociedade Brasileira de Geofísica.

This paper was prepared for presentation during the $17^{\text {th }}$ International Congress of the Brazilian Geophysical Society held in Rio de Janeiro, Brazil, $16-19$ August 2021.

Contents of this paper were reviewed by the Technical Committee of the $17^{\text {th }}$ International Congress of the Brazilian Geophysical Society and do not necessarily represent any position of the SBGf, its officers or members. Electronic reproduction or storage of any part of this paper for commercial purposes without the written consent of the Brazilian Geophysical Society is prohibited.

\section{Summary}

Recent advances in seismic imaging enables a better understanding of the subsurface. This case study illustrates the value of rejuvenating legacy seismic data using new imaging technologies to produce superior images of the subsurface that can minimize drilling risks and aid reservoir characterization in the Espirito Santo basin. Highresolution imaging around one of the prospective reservoirs was achieved by utilizing a full broadband seismic data reprocessing sequence on a streamer dataset, Full Waveform Inversion (FWI) for Velocity Model Building (VMB), and $45 \mathrm{~Hz}$ Least Squares Reverse Time migration (LS RTM) for enhanced depth imaging over the target area.

\section{Results and Conclusions}

The first key component of the imaging project was the reprocessing of the raw data through a broadband processing sequence in order to broaden the bandwidth, attenuate the multiples effectively, and ensure full data integrity (e.g., preservation of primary reflections and diffractions). The processing sequence included processes such as 3D Receiver and Source Deghosting, 3D Designature and 3D SRME.

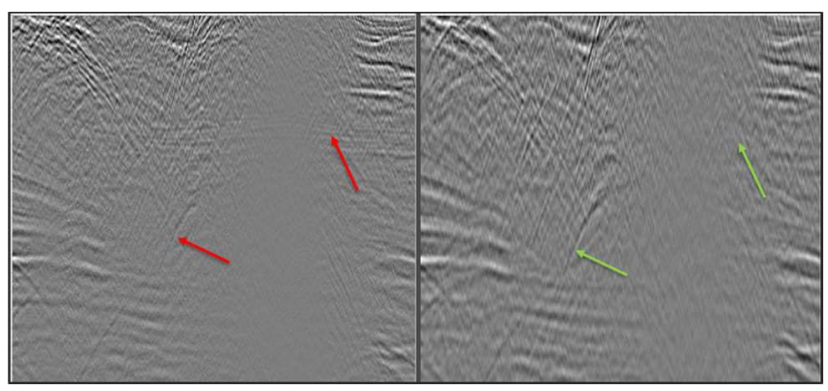

Figure 1. The left hand side shows the legacy premigration data while the right hand side shows the reprocessed data prior to migration. Red arrows show areas with residual multiples and primary attenuation, while green arrows are showing better multiple attenuation and primary preservation.

The improvements in terms of bandwidth, multiple attenuation and data integrity highlighted in Figure 1 lead to a much better input dataset for VMB and depth imaging.

Using the rejuvenated input dataset, the velocity model was build combining travel-time tomography and FWI up to $9 \mathrm{~Hz}$, and then migrated using $45 \mathrm{~Hz}$ LS RTM to produce a more reliable high-resolution image of the Turbidite reservoirs in Upper Cretaceous, which helped improving the understanding of the reservoir extension.

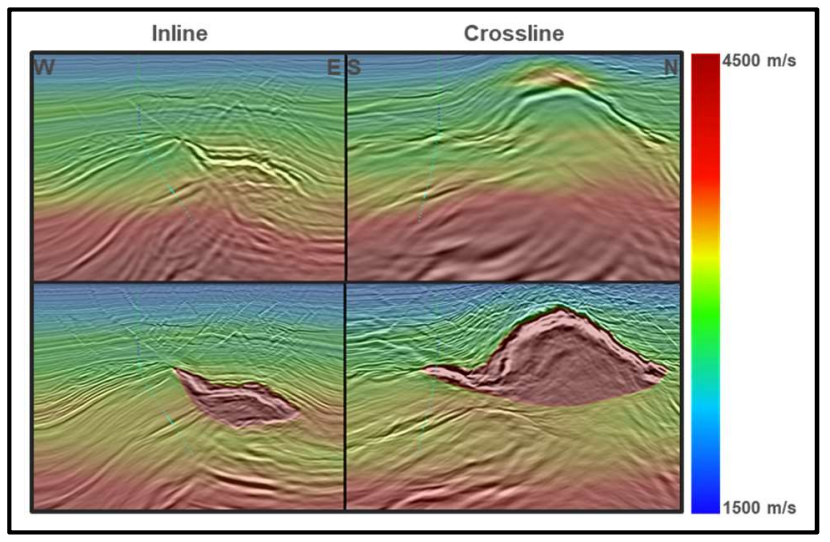

Figure 2. Comparison between legacy RTM processing (on top) versus latest LS RTM (bottom). Both datasets are overlaid with their respective final velocity model.

The comparison between legacy RTM and latest reprocessing using LS RTM (Figure 2) show that high-resolution imaging in structurally complex areas of Espirito Santo basin can be achieved by implementation of state-of-the-art technologies such as FWI and LS RTM, and a robust full data integrity broadband sequence. 